\title{
Soluciones corporativas de inteligencia de negocios en las pequeñas y medianas empresas
}

\section{Corporate business intelligence solutions in small and medium enterprises}

\author{
Esteban Ismael Cordero-Naspud \\ esteban.cordero@psg.ucacue.edu.ec \\ Universidad Católica de Cuenca, Cuenca \\ Ecuador \\ https://orcid.org/0000-0002-3442-6996 \\ Juan Carlos Erazo-Álvarez \\ jcerazo@ucacue.edu.ec \\ Universidad Católica de Cuenca, Cuenca \\ Ecuador \\ https://orcid.org/0000-0001-6480-2270 \\ Cecilia Ivonne Narváez-Zurita \\ inarvaez@ucacue.edu.ec \\ Universidad Católica de Cuenca, Cuenca \\ Ecuador \\ https://orcid.org/0000-0002-7437-9880 \\ Diego Marcelo Cordero-Guzmán \\ dcordero@ucacue.edu.ec \\ Universidad Católica de Cuenca, Cuenca \\ Ecuador \\ https://orcid.org/0000-0003-2138-2522
}

Recibido: 24 de marzo de 2020

Revisado: 09 de abril de 2020

Aprobado: 30 de abril de 2020

Publicado: 19 de mayo de 2020 


\section{RESUMEN}

El objetivo se basó en determinar el sistema inteligente que se ajuste a la operatividad de la Comercializadora "Cordero", ubicada en Azogues, provincia de Cañar, Ecuador. El método es de tipo no experimental y de enfoque mixto. Se descubrió que existe un número reducido de pymes que utilizan el sistema $\mathrm{BI}$, y las que utilizan esta tecnología la mayoría emplea la herramienta de cuadro de mando integral, la misma que se alinea en mayor porcentaje a los componentes financieros, clientes, procesos internos y aprendizaje en las organizaciones. El estudio sobre la inteligencia de negocios demuestra a la administración que esta herramienta tecnológica permite un mejor análisis de la información de forma más rápida; asimismo, mejoró significativamente la calidad de la información haciendo más confiables los reportes generados a partir de ella, brindando un soporte para la toma de decisiones en las empresas.

Descriptores: Inteligencia artificial; empresa privada; economía de mercado; toma de decisiones. (Palabras Tomadas del Tesauro UNESCO).

\section{ABSTRACT}

The objective was based on determining the intelligent system that adjusts to the operation of "Cordero" marketer, located in Azogues, Cañar province, Ecuador. The method is non-experimental and it is focused on a mixed approach. It was discovered that there is a small number of SMEs using the BI system, and most of them who apply this technology, choose the balanced scorecard tool, which is aligned in a greater percentage to the financial components, clients, internal processes and learning in organizations. The study on business intelligence shows the administration that this technological tool allows a better analysis of the information in a faster way, also it significantly improved the quality of the information, making more reliable reports and providing support for decision making in companies.

Descriptors: Artificial intelligence; private enterprises; market economy; decision making. (Words taken from the UNESCO Thesaurus).

\section{INTRODUCCIÓN}

En Ecuador al igual que todos los países, el sistema de inteligencia de negocios es una herramienta utilizada por las pymes comercializadoras de bienes, servicios y de producción con el objetivo de mejorar el rendimiento y eficiencia en su atención de acuerdo con las exigencias y creciente demanda de los usuarios, debido a que el 
mercado competitivo exige a los oferentes utilizar las tecnologías informáticas para tecnificar a las pymes. Autores como Muñoz-Hernández, Osorio-Mass \& Zúñiga-Pérez, 2016); (Vanegas-Lago \& Guerra-Cantera, 2013), señalan que la revolución tecnológica y datos informáticos que las organizaciones manejan a diario conllevan a las pymes a intensificar sus capacidades de almacenaje y procesamiento de información.

En las principales ciudades como Quito, Guayaquil, Cuenca, Ambato, etc., donde la afluencia de usuarios es mayor, se observa la introducción de sistemas informáticos que facilitan el procesamiento y atención a los usuarios, por otra parte, estos sistemas ayudan en la toma de decisiones gerenciales, a su vez proporcionan a las pymes una mejor imagen. El entorno donde trabajan los negocios en Azogues es crítico para que los empresarios brinden a los consumidores métodos de servicio eficientes, además de tomar preferencias al ritmo de desarrollo de la economía, lo que les permite lograr ventajas estratégicas para tener éxito en el sector local, por lo que Una red de información es el recurso correcto. Por ello la información debe ser de calidad para cumplir con su objetivo según (Guajardo-Cantú \& Andrade-De-Guajardo, 2014).

Distribuidora Cordero inició sus actividades comerciales el año 2015, ubicada en Azogues, provincia del Cañar-Ecuador. La actividad principal de este negocio es la comercialización de mercaderías de tipo agroalimenticios, los proveedores pertenecen a diferentes productores del cantón donde reside y del austro ecuatoriano, siendo sus clientes principales negocios mayoristas como comisariatos, markets, bares escolares y tiendas. Al tener una gran cantidad de existencias de productos, se produce la dificultad de gestionar líneas de productos como la identificación de la adquisición de productos, compradores, la rotación de inventarios y, sobre todo, la realización de la venta de bienes, lo que restringe el negocio a la competencia del sector local; además carece de análisis y procesamiento rápido y preciso de información que poseen en la distribuidora, Por lo tanto, el conocimiento y uso de las tecnologías enfocado al ámbito empresarial genera ventajas competitivas ayudando a las organizaciones agregar valor y reforzar su estadía en el mercado comercial. 


\section{Referencial teórico}

\section{Los sistemas de información}

Los sistemas de información (SI), en las pymes forman parte de las herramientas básicas para el desarrollo operativo y análisis de la situación de la organización, agrupa los recursos mediante tres funciones definidas como, recibir datos informativos de fuentes internas y externas, interviene y opera los datos recolectados y finalmente imparte la información procesada a los usuarios con el propósito de desarrollar estrategias que ayuden en el mejoramiento de la empresa. Al respecto, AbregoAlmazán, Sánchez-Tovar \& Medina-Quintero (2017), sostienen que "Los sistemas de información (SI) son uno de los componentes más relevantes del entorno actual de negocios, que ofrecen grandes oportunidades de éxito para las pymes".

Turban, Volonino \& Wood, (2013) expresan que "Los SI utilizan equipos de cómputo, bases de datos, software, procedimientos, modelos para el análisis y procesos administrativos para la toma de decisiones". Estos sistemas para su operatividad requieren de estos elementos básicos, en los cuales se incrementan nuevas tecnologías que permiten obtener mayor control del entorno operativo y productivo del ser humano.

Desde el punto de vista empresarial, los sistemas de información facilitan la recopilación, administración, procesamiento, análisis y creación de estrategias con la finalidad de cumplir con los objetivos y metas organizacionales, cabe puntualizar que los SI, comprenden tanto equipos informáticos, tecnológicos, recursos materiales, económicos y humanos que se utilizan para el cumplimiento operacional, por otra parte, un factor importante de resaltar es el conocimiento en el manejo de los sistemas tecnológicos de información, la identificación del sistema adecuado para la empresa y el uso correcto del sistema facilitarán obtener resultados informativos eficientes, a tiempo y confiables.

Respecto a los datos, Roo-Huerta \& Boscán-Romero (2012). mencionan que la base de datos, "Es el almacenaje de diferentes temas o información para ser analizada de forma conjunta o independiente y facilita efectuar consultas, análisis y presentación de 
información concreta" (p. 553). Finalmente, la gestión del conocimiento como manifiestan (Reyes-Dixson \& Nuñez-Maturel, 2015), constituye parte elemental para el manejo de las organizaciones mediante el uso de un sistema integrado que abarca contenidos informáticos, datos, tecnologías y conocimientos para el manejo empresarial.

\section{Sistema inteligente de negocios}

Es considerado un instrumento que forma parte de los activos tangibles utilizado por las pymes para agregar valores y construir estrategias competitivas en los mercados, enmarca otras herramientas para crear el business intelligence $(\mathrm{BI})$ como herramienta para la toma de decisiones. (Vanegas-Lago \& Guerra-Cantera, 2013), en referencia al Sistema de Inteligencia de Negocios opinan que permite capturar, almacenar, procesar, analizar y mostrar de manera eficiente la información operativa y administrativa de la empresa con fines de comprensión y uso de estrategias. Por su parte (MedinaChicaiza, Chiliquinga-Vejar \& Ortiz-Barba (2016). sostienen que "Es el conjunto de tecnologías que facilitan a las pymes sintetizar la información con el objetivo de asumir decisiones correctas y competitivas" (p. 370).

En efecto, esta herramienta disponible en el mercado que funciona gracias a las ventajas que ofrece el internet y las tecnologías de la información, constituye un tema amplio de uso, exploración y explotación en todas las dimensiones de trabajo comercial, productivo y de servicio. Con respecto al campo de las ciencias administrativas y empresariales, muchos académicos han publicado sus investigaciones relevantes para este análisis sobre programas de inteligencia empresarial, programas y métodos de información gerencial para el desarrollo competitivo de las pymes, que requieren el aporte de experiencia en el tema para para posteriormente derivar los resultados.

Por consiguiente, (Ramírez \& Vega, 2015), identificaron "las diferentes fases con lo cual los procedimientos informativos y la invención se modifican y forman parte del factor de crecimiento de las pymes" (p. 201). Estos conocimientos previos utilizados en el contorno de los negocios permiten obtener la información fundamental para intervenir 
mediante la elaboración y uso de estrategias que actúan con la aplicación de tecnologías informáticas.

Por otro lado, (Gutiérrez-Camelo, Devia-Llanos \& Tarazona-Bermúdez, 2016), presentan los resultados del análisis efectuado a 90 pymes de Colombia del área tecnológica con relación a la cantidad de compañías que utilizan métodos de inteligencia como estrategia de apoyo para las decisiones de gerencia, obteniendo como resultado que el $96 \%$ de las organizaciones encuestadas si tienen conocimiento respecto a los $\mathrm{BI}$ y sobre los beneficios que se pueden conseguir mediante el uso de esta herramienta, siendo los productos más destacados aquellos relacionados como los sistemas gestores de control de mercaderías y clientes sin embargo solo el $34,4 \%$ de pymes posee en su empresa un sistema inteligente, con respecto al grupo de negocios que no posee aun este instrumento se debe a los altos costos económicos para la instalación de la estructura tecnológica.

Así mismo, (González, Palacios \& Perea, 2017), efectuaron un estudio sobre la relación entre la Inteligencia de Negocios y su rol en la agilidad organizacional. La metodología que aplicaron se fundamentó en la revisión sistemática de literatura de Business Intelligence $(\mathrm{BI})$ y agilidad organizacional a 90 artículos publicados en idioma inglés y español en revistas científicas, previo a la selección sobre temas en relación con mercadeo estratégico, sistemas de información y gestión empresarial llegando a analizar un total de 62 artículos concluyendo que "no existe un consenso sobre si el éxito de los productos $\mathrm{BI}$ y la $\mathrm{AO}$ son variables independientes, o si alguna es variable dependiente de la otra. Sin embargo, es creciente la aceptación de una relación positiva entre ambas".

Por su parte, (Cordero-Guzmán \& Rodríguez-López, 2017), en la investigación efectuada sobre "la inteligencia de negocios: una estrategia para la gestión de las pymes productivas", determinaron que las organizaciones ubicados en Ecuador, cantón La Troncal perteneciente a la provincia del Cañar, en un total de 130 pymes analizadas tienen la predisposición de financiar una aplicación inteligente de negocios. (CorderoGuzmán \& Rodríguez-López, 2017), expresan que "El 81\% de organizaciones tienen la 
posibilidad menor a los $\$ 5.000$ Usd., un $13 \%$ dispone entre $\$ 7.000,00$ a $\$ 10.000,00$ Usd., solo un 6\% dispone de más de 10000 dólares". Esta información evidencia que los recursos económicos son limitados lo que indica que la disponibilidad del presupuesto para la inversión en tecnología BI es baja, con relación a la importancia y ventajas estratégicas que ofrece el sistema. En otro orden, (Da Silva, Pastor \& Pastor, 2014), efectuaron el estudio con relación al

Uso del Cuadro de Mando Integral como Instrumento de Medición para comparar los Modelos de Excelencia en Gestión" con la finalidad de realizar un "Análisis comparativo de los modelos de excelencia en gestión European Foundation Quality Management (EFQM), Baldridge Performance Excellence Program (BPEP) y Modelo de Excelencia da Gestión (MEG), utilizando como instrumento de medición las perspectivas del Cuadro de Mando Integral (CMI).

El método que utilizaron fue el estudio de contenido, mediante la búsqueda y puntualización de razonamientos y subcriterios de cada modelo y sus concordancias con las configuraciones del CMI. Las soluciones que obtuvieron del análisis muestran temas homogéneos entre los 3 tipos examinados (EFQM, BPEP y MEG), así como un vínculo entre cada sub-enfoque y sus propias valoraciones a las facetas del $\mathrm{CMI}$, siendo factible efectuar una ponderación de aporte porcentual de cada enfoque individual como punto de vista con relación al Cuadro de Mando Integral CMI.

\section{Ventajas de la inteligencia de negocios}

Debido a la amplia gama de servicios que ofrece permite conocer los niveles de ingresos que una empresa obtiene en diferentes periodos contables, además medir el nivel de ventas, conocer cuál es el producto de mayor venta a nivel general o de manera focal, en fin, ayuda a conocer muchos parámetros en tiempos reducidos solo con aplicar las fórmulas o indicadores para cada variable que se desea conocer. En este sentido, (Herazo, 2018) menciona algunas ventajas que se adquieren con este modelo: 
- Examinar datos de varias fuentes bases de datos o en la nube, lo que deja correlacionar datos y tomar decisiones más convenientes.

- Tener acceso a tableros de control desde el PC smartphone para acceder a los datos que se requieran desde cualquier sitio donde nos encontremos solo con la condición de disponer de internet.

Por su parte, (Coronel, Morris \& Rob, 2011) manifiestan que otros beneficios que ofrecen la inteligencia de negocios es el ahorro de tiempo para encontrar información específica, además de la formulación de gráficas e indicadores para conocimiento gerencial en corto tiempo. Por otra parte, con la información ingresada a la base de datos que realiza cada departamento, los administradores pueden determinar cuáles son las debilidades o puntos críticos que generan mayores gastos con el propósito de aplicar estrategias adecuadas para reducir dichos gastos. Ayuda a estipular metas realistas mediante la comparación de los resultados financieros históricos y actuales o también mediante la comparación con los resultados de negocios similares, solo así se puede elaborar proyecciones posibles de alcanzar.

\section{Componentes de la inteligencia de negocios}

Los sistemas (BI) organizan la compilación de datos, el almacenamiento de dicha información y la gestión del conocimiento con instrumentos analíticos para entender y dilucidar información compleja que permita procesar y obtener resultados de forma eficiente a fin de que la secuencia de las actividades empresariales continúe su curso habitual. Los principales componentes que se encuentran en estos sistemas están las fuentes de información, ETL (extracción transformación), minería de datos, aplicaciones analíticas, bandeja de datos, Data Warehouse que a su vez contiene subcomponentes. Las fuentes de información están integradas por el conjunto de datos relacionados a un tema específico y común entre sí que se almacena ordenadamente para ser utilizado cuando el usuario cree conveniente utilizar la información, por lo tanto, debe ser almacenado mediante registros definidos e identificables. (Reyes-Dixson \& NuñezMaturel, 2015), expresan "El componente fuente de datos define las fuentes que se 
utilizarán en la obtención de los datos que utiliza el sistema" (p. 68). La preparación de los datos está integrada por dos subáreas, en la una se almacena la información mientras que en el otro componente se efectúa el conjunto de procesos. El componente extract, transform and Load (ETL), como lo expresa (Sherman, 2014) son las fases de tratamiento que se utilizan mediante las diversas fuentes de datos como las redes sociales, gestión del cliente, customer relation's management CRM, Enterprise Resource Planning ERP, etc., para cargar el Data Warehouse con información. La extracción de datos se refiere a la obtención seleccionada de la información que se encuentra en la base de datos que se relaciona con un tema específico. La transformación comprende la información inicial extraída para posteriormente ser transformada en un formato específico conservando su conjunto original, la transformación permite efectuar operaciones para validar, calcular, codificar, filtrar y duplicar mediante respaldo de seguridad la información. La carga hace referencia a los resultados obtenidos del procesamiento de datos, es decir es el conocimiento que se obtiene luego del análisis de la información de la base de datos.

\section{Data Warehouse}

Conocido como Almacén de datos son archivos de información recopilada de varias fuentes y durante varias épocas para ser usadas en cualquier tiempo con el objetivo de posteriormente ser utilizada en la empresa, esta herramienta contribuye para una toma de decisiones, por otro lado, (Camargo-Vega, Joyanes-Aguilar \& Giraldo-Marín, 2016), mencionan que previo al almacenamiento de la información en este sistema, estos deben ser tratados de manera especial, es decir se debe utilizar el ETL que quiere decir (extraer, limpiar, transformar y cargar) todos los valores de forma secuencial. 
Revista Arbitrada Interdisciplinaria KOINONIA

Año V. Vol V. N${ }^{\circ}$ 10. Julio - Diciembre 2020

Hecho el depósito de Ley: FA2016000010

ISSN: 2542-3088

FUNDACIÓN KOINONIA (F.K). Santa Ana de Coro. Venezuela.

Esteban Ismael Cordero-Naspud; Juan Carlos Erazo-Álvarez; Cecilia Ivonne Narváez-Zurita; Diego Marcelo Cordero-Guzmán

\section{Herramientas de análisis, consulta y visualización}

\section{Minería de datos}

A criterio de (Vásquez, 2015), la minería de datos es un acopio de instrumentos de evaluación de información que mediante la determinación de patrones permite obtener de manera concreta lo que se requiere saber sobre un proceso específico o general empresarial, es básicamente necesaria cuando se requiere ejecutar una decisión. Así mismo, (Uvidia-Fassler, Cisneros-Barahona, Méndez-Naranjo \& Villa-Yánez, 2019), manifiestan que la minería de datos comprende la extracción de conocimiento a partir de grandes cantidades de datos sobre los cuales se emplean herramientas inteligentes con el objetivo de extraer la información específica sobre la cual se requiere desarrollar un trabajo específico. Es decir que la extracción se centra en la toma de una porción del todo que contiene la minería de datos.

\section{El cuadro de mando integral}

Presentado por (Kaplan \& Norton, 2009) utiliza varios indicadores que permiten tener un panorama general de la organización, además del conocimiento preliminar sobre los activos que posee la organización a través de la medición de los valores con el uso de los indicadores que ofrece este sistema. Los valores intangibles este modelo facilita incluir en la empresa giran en base al conocimiento para clarificar la visión y estrategias organizacionales, se basa en la comunicación de los objetivos trascendentales para incrementar la comunicación dentro de los empleados además de orientar las iniciativas estratégicas con el objetivo de mejorar la apropiación de determinaciones acertadas. Los indicadores que este modelo se refiere a, perspectiva financiera, interna, de aprendizaje y del cliente.

\section{Las decisiones en las organizaciones}

Por consiguiente, Vercellis (2009) define que "Una decisión es una elección que se toma entre varias alternativas" (p. 10). Rifai (2015) expresa que "la toma de decisiones es el proceso de identificación de un problema u oportunidad y la selección de una 
alternativa de acción entre opciones, por lo tanto, es vital saber decidir bien"(p. 4), (Koontz, Weihric, \& Cannice, 2012) expresan que "Es el núcleo de la planeación, y se define como la selección de un curso de acción entre varias alternativas" (p. 152). El decidir sobre cómo actuar y qué estrategias aplicar son una temática siempre analizada cuando se realiza la distribución de la estructura de las pymes, debido a que de ello depende en parte la asignación de las actividades operativas, responsabilidad en el desempeño y grupal de trabajo y grado de autoridad de cada departamento que forma parte de la empresa.

\section{Sistemas de información y decisiones}

Con respecto a los sistemas de información y las decisiones a criterio de Laudon \& Laudon (2012) "Son el conjunto de componentes interrelacionados cuyo fin es recolectar, restaurar, procesar, almacenar, distribuir y seleccionar datos que facilitan efectuar decisiones asertivas para controlar a la empresa" (p. 24). El decidir sobre cómo actuar y qué estrategias aplicar son una temática siempre analizada cuando se realiza la distribución de la estructura de las pymes, debido a que de ello depende en parte la asignación de las actividades operativas, responsabilidad en el desempeño y grupal de trabajo además del grado de autoridad de cada departamento que forma parte de la empresa.

La importancia de las decisiones correctas, efectivamente, previo a una toma de decisiones se requiere la suficiente información, misma que debe ser basada en datos certeros para que los administradores puedan optar por un correcto criterio de decidir. Cuando la información que disponga el gerente es escasa o se encuentre basada en datos poco confiables, la toma de decisiones se convierte en limitada. Otras causas que limitan una decisión oportuna tienen que ver con el entorno externo del negocio como por ejemplo la situación política, económica, el medio cultural y social, el mercado local, criterios de moda y preferencias del comprador, entre otras variables. 


\section{Etapas del sistema de información inteligente}

Para efectuar una buena toma de decisiones existen etapas que el administrador o gerente debe considerar tales como: Identificación del problema, primeramente, se requiere conocer cuál es la causa que altera o no permite el cumplimiento del flujo de proceso operativo, limitando el cumplimiento de las metas. Tipificación de los criterios de decisión, esta fase tiene como objetivo canalizar la estrategia más conveniente para solucionar el problema mediante la intervención de la opinión de los individuos responsables de los departamentos de la empresa donde se suscitó el problema a fin de conocer cuáles son los procedimientos que se siguieron y cuáles fueron los que se omitieron, ya que son necesarios para decidir correctamente.

Asignación de criterios, expresados en la identificación que es argumentada por los jefes de áreas departamentales deben ser priorizados de manera correcta, es decir van desde el más importante al que menos afectación ha causado en la problemática encontrada. Desarrollo de alternativas, significa en elaborar diferentes estrategias o alternativas para dar solución al problema.

Análisis de alternativas, en esta etapa, como lo señala (Teruel (2019) el responsable de tomar las decisiones en la organización deberá efectuar un análisis minucioso a las alternativas existentes que permitan dar fin a las problemáticas circunstanciales. Por lo indicado es necesario el estudio de las áreas fuertes y débiles que presentan cada alternativa como solución al problema. de la eficacia de la decisión tomada, consiste en examinar después la alternativa adecuada para dar solución al problema con el propósito de conocer si éste se solucionó.

\section{MÉTODO}

La metodología que se utilizó en el presente estudio es de tipo no experimental, debido a que no se efectuó manipulación de variables, por el contrario, se recurrió al enfoque mixto, donde se empleó los métodos cualitativo y cuantitativo además de la aplicación de otros métodos como el sistémico, inductivo, deductivo, analítico y sintético. El alcance de esta investigación fue descriptivo - explicativo debido a que las variables 
planteadas se describieron y explicaron en el presente documento. Con respecto a la finalidad, esta investigación fue transversal, la recolección de los datos se realizó en un solo momento y se explicó sus relaciones e incidencias (Saquicela-Rodas, ErazoÁlvarez, \& Narváez-Zurita, 2019).

\section{Universo de estudio y tratamiento muestral}

Las entrevistas se aplicaron a pymes de Azogues, provincia del Cañar, en base a la cantidad de negocios comerciales y de servicios según el catastro que posee el (Instituto Nacional de Estadistica y Censos, 2019), indica que la ciudad en mención a diciembre de 2019 posee 9.798 negocios activos, de los cuales 55 son Comercializadoras y distribuidoras de víveres al por mayor y menor. Por lo tanto, como el universo de estudio es reducido, para la presente investigación se procedió a efectuar la entrevista a un representante administrativo de cada empresa de las 55 comercializadoras identificadas en la ciudad de Azogues.

\section{Tratamiento estadístico de la información}

Los datos que se extrajeron de los cuestionarios a través del empleo de los formularios de Google Forms (https://docs.google.com/forms/u/0/) fueron tabulados en el programa estadístico SPSS VERSIÓN 23. Las respuestas recogidas se contabilizaron y agruparon conforme a los componentes planteados y se graficaron mediante el empleo del programa Excel perteneciente al paquete de Office 365, mediante la estadística descriptiva.

\section{RESULTADOS}

Las dos primeras preguntas se tabularon independientemente, se requiere determinar la cantidad de pymes que poseen un (SI) de negocios y el tipo de herramienta con el que trabajan. Luego se clasificaron los cuatro componentes con los subcomponentes que forman parte de un sistema inteligente y bajo esta información se procesó las respuestas para determinar los beneficios que ofrecen los sistemas inteligentes. 
La ciudad de Azogues al momento registra 55 pymes comercializadoras de productos de las cuales el $22 \%$ sí posee un sistema inteligente, mientras que el $78 \%$ carece de esta herramienta que ayude en la toma de decisiones gerenciales.

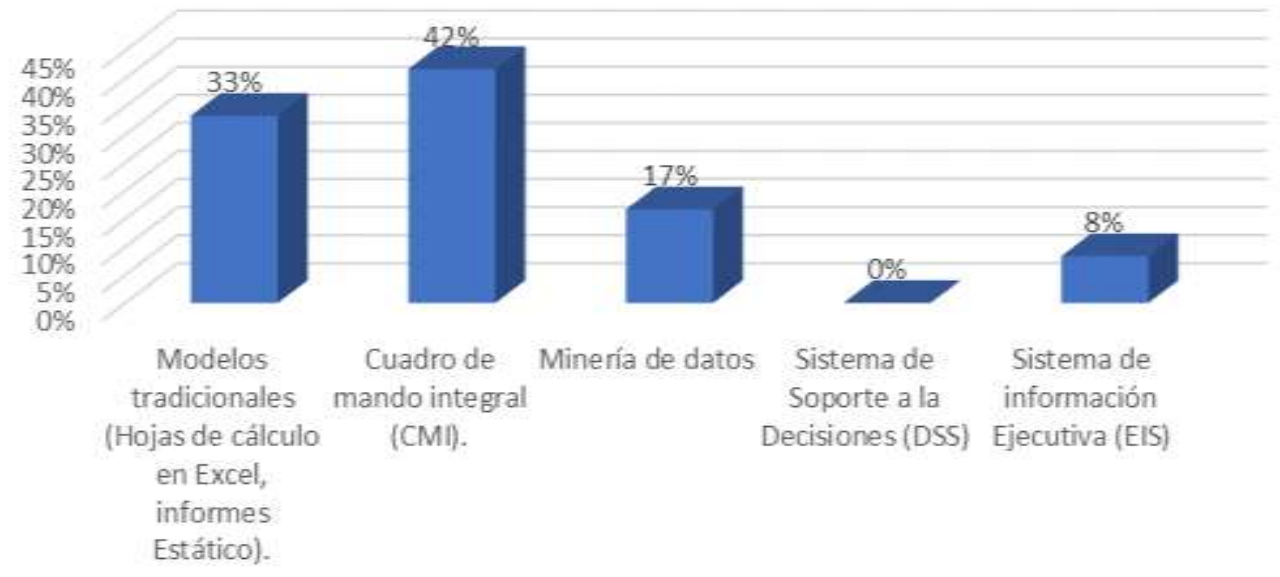

Figura 1. Sistemas inteligentes utilizados. Fuente: Investigación de campo

El sistema de mayor uso por parte de las pymes de este sector es el CMI con el $42 \%$, los Modelos Tradicionales el 33\%, Minería de Datos 17\% y EIS 8\%, con relación a OLAP $\mathrm{u}$ otros sistemas ninguna empresa hace referencia, por lo tanto, a partir de esta información las siguientes variables se tabularon en base a la información que proporcionaron 12 pymes. 
Revista Arbitrada Interdisciplinaria KOINONIA

Año V. Vol V. $\mathrm{N}^{\circ} 10$. Julio - Diciembre 2020

Hecho el depósito de Ley: FA2016000010

ISSN: 2542-3088

FUNDACIÓN KOINONIA (F.K). Santa Ana de Coro. Venezuela.

Esteban Ismael Cordero-Naspud; Juan Carlos Erazo-Álvarez; Cecilia Ivonne Narváez-Zurita; Diego Marcelo Cordero-Guzmán

\begin{tabular}{|c|c|c|c|c|c|c|c|c|c|c|}
\hline \multirow{3}{*}{ Sistema } & \multicolumn{10}{|c|}{ Financiera } \\
\hline & \multicolumn{3}{|c|}{ Rentabildad } & \multicolumn{3}{|c|}{ Ingres os } & \multicolumn{3}{|c|}{ Eiciencia costos } & \multirow{2}{*}{$\begin{array}{c}\text { Total } \\
\%\end{array}$} \\
\hline & $\begin{array}{l}\text { Exce } \\
\text { lente }\end{array}$ & $\begin{array}{c}\text { Muy } \\
\text { bueno }\end{array}$ & Bueno & $\begin{array}{l}\text { Exce } \\
\text { lente }\end{array}$ & $\begin{array}{c}\text { Muy } \\
\text { bueno }\end{array}$ & Bueno & $\begin{array}{l}\text { Exce } \\
\text { lente }\end{array}$ & $\begin{array}{c}\text { Muy } \\
\text { bueno }\end{array}$ & Bueno & \\
\hline $\begin{array}{l}\text { Modelos tradicionales (Hojas de calculo } \\
\text { en Excel, informes Estático). }\end{array}$ & $8 \%$ & $4 \%$ & $4 \%$ & $6 \%$ & $4 \%$ & $6 \%$ & $6 \%$ & $6 \%$ & $4 \%$ & $15 \%$ \\
\hline Cuadro de mando integral (CM). & $4 \%$ & $58 \%$ & $8 \%$ & $8 \%$ & $44 \%$ & $17 \%$ & $8 \%$ & $52 \%$ & $10 \%$ & $69 \%$ \\
\hline Minería de datos & $0 \%$ & $8 \%$ & $0 \%$ & $0 \%$ & $6 \%$ & $2 \%$ & $0 \%$ & $4 \%$ & $4 \%$ & $8 \%$ \\
\hline Sistema de información Ejecutiva (EIS) & $2 \%$ & $6 \%$ & $0 \%$ & $2 \%$ & $6 \%$ & $0 \%$ & $2 \%$ & $6 \%$ & $0 \%$ & $8 \%$ \\
\hline \multirow{4}{*}{ Sistema } & $13 \%$ & $75 \%$ & $12 \%$ & $15 \%$ & $60 \%$ & $25 \%$ & $15 \%$ & $67 \%$ & $17 \%$ & $100 \%$ \\
\hline & \multicolumn{10}{|c|}{ Clientes } \\
\hline & \multicolumn{3}{|c|}{ Incremento clientes } & \multicolumn{3}{|c|}{ Satisfac ción clientes } & \multicolumn{3}{|c|}{$\begin{array}{c}\text { Más partic ipación en } \\
\text { mercado }\end{array}$} & \multirow{2}{*}{$\begin{array}{c}\text { Total } \\
\%\end{array}$} \\
\hline & $\begin{array}{l}\text { Exce } \\
\text { lente }\end{array}$ & $\begin{array}{l}\text { Muy } \\
\text { bueno }\end{array}$ & Bueno & celen & $\begin{array}{c}\text { Muy } \\
\text { bueno }\end{array}$ & Bueno & tcelen & $\begin{array}{c}\text { Muy } \\
\text { bueno }\end{array}$ & Bueno & \\
\hline $\begin{array}{l}\text { Modelos tradicionales (Hojas de cálculo } \\
\text { en Excel, informes Estático). }\end{array}$ & $4 \%$ & $6 \%$ & $6 \%$ & $4 \%$ & $6 \%$ & $6 \%$ & $4 \%$ & $6 \%$ & $6 \%$ & $15 \%$ \\
\hline Cuadro de mando integral (CM). & $12 \%$ & $35 \%$ & $23 \%$ & $10 \%$ & $37 \%$ & $23 \%$ & $6 \%$ & $46 \%$ & $17 \%$ & $69 \%$ \\
\hline Mineria de datos & $0 \%$ & $6 \%$ & $2 \%$ & $0 \%$ & $4 \%$ & $4 \%$ & $0 \%$ & $6 \%$ & $2 \%$ & $8 \%$ \\
\hline Sistema de inform ación Ejecutiva (EIS) & $2 \%$ & $6 \%$ & $0 \%$ & $2 \%$ & $6 \%$ & $0 \%$ & $2 \%$ & $6 \%$ & $0 \%$ & $8 \%$ \\
\hline \multirow[t]{4}{*}{ Total } & $17 \%$ & $52 \%$ & $31 \%$ & $15 \%$ & $52 \%$ & $33 \%$ & $12 \%$ & $63 \%$ & $25 \%$ & $100 \%$ \\
\hline & \multicolumn{10}{|c|}{ Procesos } \\
\hline & \multicolumn{3}{|c|}{ Mejor logistica } & \multicolumn{3}{|c|}{ Cadena de suministros } & \multicolumn{3}{|c|}{ Control de gestión } & \multirow{2}{*}{$\begin{array}{c}\text { Total } \\
\%\end{array}$} \\
\hline & $\begin{array}{l}\text { Exce } \\
\text { lente }\end{array}$ & $\begin{array}{c}\text { Mury } \\
\text { bueno }\end{array}$ & Bueno: & celen & $\begin{array}{c}\text { Muy } \\
\text { bueno }\end{array}$ & Bueno & tcelen & $\begin{array}{c}\text { Muy } \\
\text { bueno }\end{array}$ & Bueno & \\
\hline $\begin{array}{l}\text { Modelos tradicionales (Hojas de cálculo } \\
\text { en Excel, informes Estático). }\end{array}$ & $4 \%$ & $4 \%$ & $8 \%$ & $4 \%$ & $8 \%$ & $4 \%$ & $4 \%$ & $6 \%$ & $6 \%$ & $15 \%$ \\
\hline Cuadro de mando integral (CM). & $4 \%$ & $60 \%$ & $6 \%$ & $8 \%$ & $44 \%$ & $17 \%$ & $4 \%$ & $58 \%$ & $8 \%$ & $69 \%$ \\
\hline Mineria de datos & $2 \%$ & $4 \%$ & $2 \%$ & $2 \%$ & $4 \%$ & $2 \%$ & $4 \%$ & $4 \%$ & $0 \%$ & $8 \%$ \\
\hline Sistema de información Ejecutiva (EIS) & $2 \%$ & $6 \%$ & $0 \%$ & $2 \%$ & $2 \%$ & $4 \%$ & $2 \%$ & $4 \%$ & $2 \%$ & $8 \%$ \\
\hline \multirow[t]{4}{*}{ Total } & $12 \%$ & $73 \%$ & $15 \%$ & $15 \%$ & $58 \%$ & $27 \%$ & $13 \%$ & $71 \%$ & $15 \%$ & $100 \%$ \\
\hline & \multicolumn{10}{|c|}{ Aprendizaje } \\
\hline & \multicolumn{3}{|c|}{ Uso de TIC's } & \multicolumn{3}{|c|}{ Capacitación pers onal } & \multicolumn{3}{|c|}{ Sistema de gestión } & \multirow{2}{*}{$\begin{array}{c}\text { Total } \\
\%\end{array}$} \\
\hline & $\begin{array}{l}\text { Exce } \\
\text { lente }\end{array}$ & $\begin{array}{c}\text { Muy } \\
\text { bueno }\end{array}$ & Bueno: & celen & $\begin{array}{c}\text { Mury } \\
\text { bueno }\end{array}$ & Bueno & tcelen & $\begin{array}{c}\text { Muy } \\
\text { bueno }\end{array}$ & $\overline{\text { Bueno }}$ & \\
\hline $\begin{array}{l}\text { Modelos tradicionales (Hojas de cálculo } \\
\text { en Excel. informes Estático). }\end{array}$ & $4 \%$ & $6 \%$ & $6 \%$ & $4 \%$ & $4 \%$ & $8 \%$ & $4 \%$ & $8 \%$ & $4 \%$ & $16 \%$ \\
\hline Cuadro de mando integral (CM). & $4 \%$ & $54 \%$ & $12 \%$ & $6 \%$ & $40 \%$ & $23 \%$ & $10 \%$ & $35 \%$ & $25 \%$ & $69 \%$ \\
\hline Mineria de datos & $4 \%$ & $4 \%$ & $0 \%$ & $4 \%$ & $0 \%$ & $4 \%$ & $2 \%$ & $2 \%$ & $4 \%$ & $8 \%$ \\
\hline Sistema de información Ejecutiva (EIS) & $8 \%$ & $0 \%$ & $0 \%$ & $2 \%$ & $4 \%$ & $2 \%$ & $0 \%$ & $6 \%$ & $2 \%$ & $8 \%$ \\
\hline Total & $19 \%$ & $63 \%$ & $18 \%$ & $15 \%$ & $48 \%$ & $37 \%$ & $15 \%$ & $50 \%$ & $35 \%$ & $100 \%$ \\
\hline
\end{tabular}

Figura 2. Componentes y subcomponentes del sistema inteligente de negocios. Fuente: Investigación de campo 
Las comercializadoras tienen un factor común que es la obtención de rentabilidad para los accionistas, el dinero es su prioridad, por lo tanto, para llegar a este punto se necesita conocer el nivel de ingresos y costos que permiten generar los SI que manejan las pymes. El $69 \%$ utiliza el CMI, el 15\% el modelo tradicional que sirve para el control contable, el 8\% Minería de Datos, el 8\% el Sistema de información Ejecutiva (EIS).

El indicador relacionado a clientes tiene tres subcomponentes a valuar, el primero relacionado con el incremento de clientes, el segundo analiza la satisfacción de los clientes, el tercero permite examinar el nivel de participación en el mercado y el cuarto se enfoca al análisis de la imagen de marca. De acuerdo con los resultados se determinó que el CMI y (EIS), son los que mayores ventajas ofrecen. Los resultados obtenidos son Cuadro de Mando Integral CMI aporta con el 69\%, el Sistema de Información Ejecutiva EIF con el 8\%, Minería de Datos con 8\% y el Modelo Tradicional el $15 \%$.

La perspectiva de los procesos internos tiene como propósito optimizar la cadena de valor de la empresa, por lo tanto, debe ser estructurada de acuerdo con las necesidades de cada organización, bajo esta conceptualización y según los resultados el Cuadro de Mando Integral CMI aporta con el 69\%, el Sistema de Información Ejecutiva EIF con el $8 \%$, Minería de Datos con $8 \%$ y el Modelo Tradicional el $15 \%$.

Esta perspectiva se hace referencia al manejo de recursos humanos y tecnológicos, según la capacitación, conocimiento y dominio sobre el SI, los resultados esperados se conseguirán. Bajo este enfoque y con los resultados obtenidos optimizan el uso de la tecnología a través de capacitaciones del personal, infraestructura y aprovechamiento de la herramienta las pymes que poseen el Cuadro de Mando Integral (CMI) obteniendo un aporte del 69\%, el Sistema de Información Ejecutiva EIS con el 8\%, Minería de Datos con $8 \%$ y el Modelo Tradicional el $15 \%$.

En resumen, según los modelos inteligentes empleados por pymes comercializadoras y en base a las descripciones, características e indicadores de algunos sistemas 
inteligentes, el modelo o herramienta más usada es el Cuadro de Mando Integral (CMI), conocido también como "Balanced Scorecard".

\section{PROPUESTA}

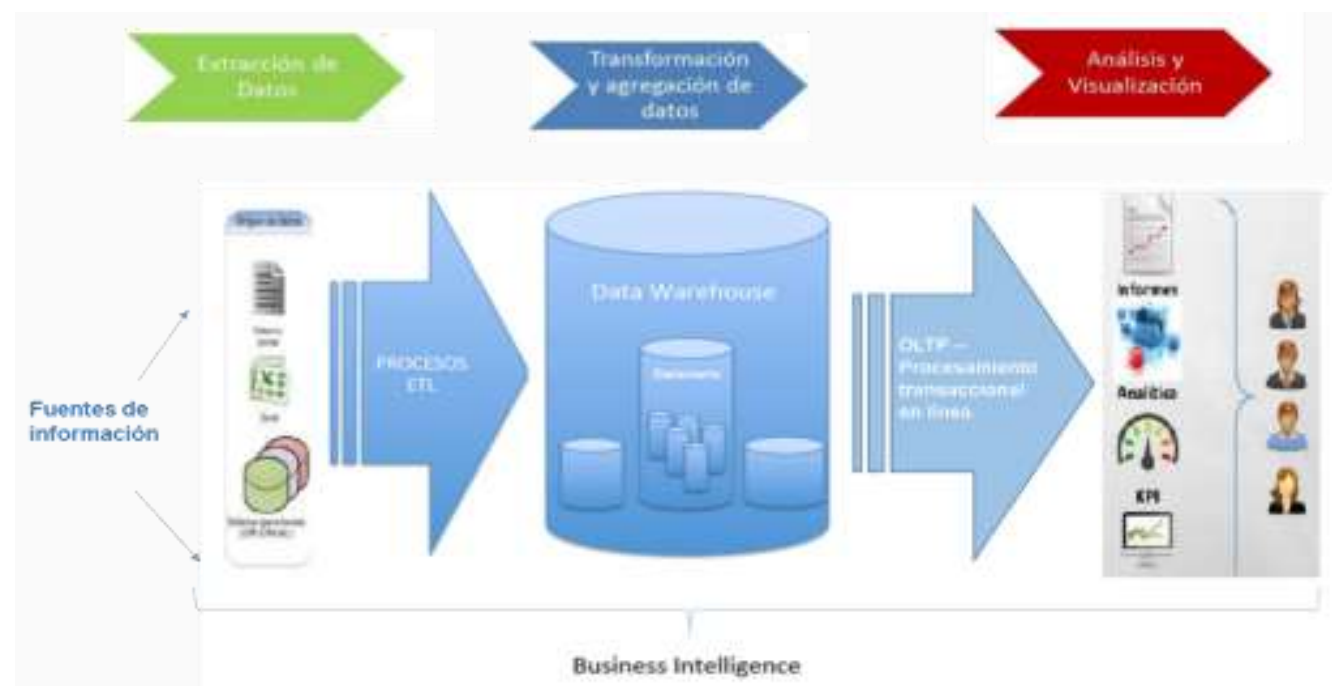

Figura 3. Propuesta de BI para pymes. Fuente: Elaboración Propia

Con el propósito de mejorar la gestión interna de Comercializadora Cordero, se propone un Sistema BI enfocado al departamento de ventas donde se realiza algunas consultas utilizando los programas Power $\mathrm{BI}$ y Microsoft Access, en los cuales se visualiza: Monto de ventas acumuladas en el periodo, monto de ventas por el primer trimestre, producto más vendido representado en valor monetario y mes donde existe más rotación de un determinado producto; cabe mencionar que para realizar este prototipo de $\mathrm{BI}$ la comercializadora Cordero proporciona datos de ventas del periodo 2018; con este modelo de BI se pretende demostrar a la organización que se puede mejorar la competitividad, calidad de información y generar conocimiento que permita tomar mejores decisiones. 


\section{Hechos y Dimensiones}

Antes de realizar el procedo de $\mathrm{BI}$ se define la tabla de hechos y dimensiones, mismo que se ha realizado siguiendo esquema de estrella. El esquema de estrella consta de una tabla de hechos (central) donde contiene los datos para el análisis, rodeada de las tablas de dimensiones; que en este caso los hechos son las ventas y las dimensiones son los clientes, productos y fechas; a continuación, la información de las transacciones de la empresa se encuentra en hojas de cálculo dispersas, los cuales se cargaran a una sola base de datos puede ser en Microsoft Excel o en otro programa como Microsoft SQL Server Management Studio para su almacenamiento y posteriormente acceder a ellos en forma rápida y estructurada.

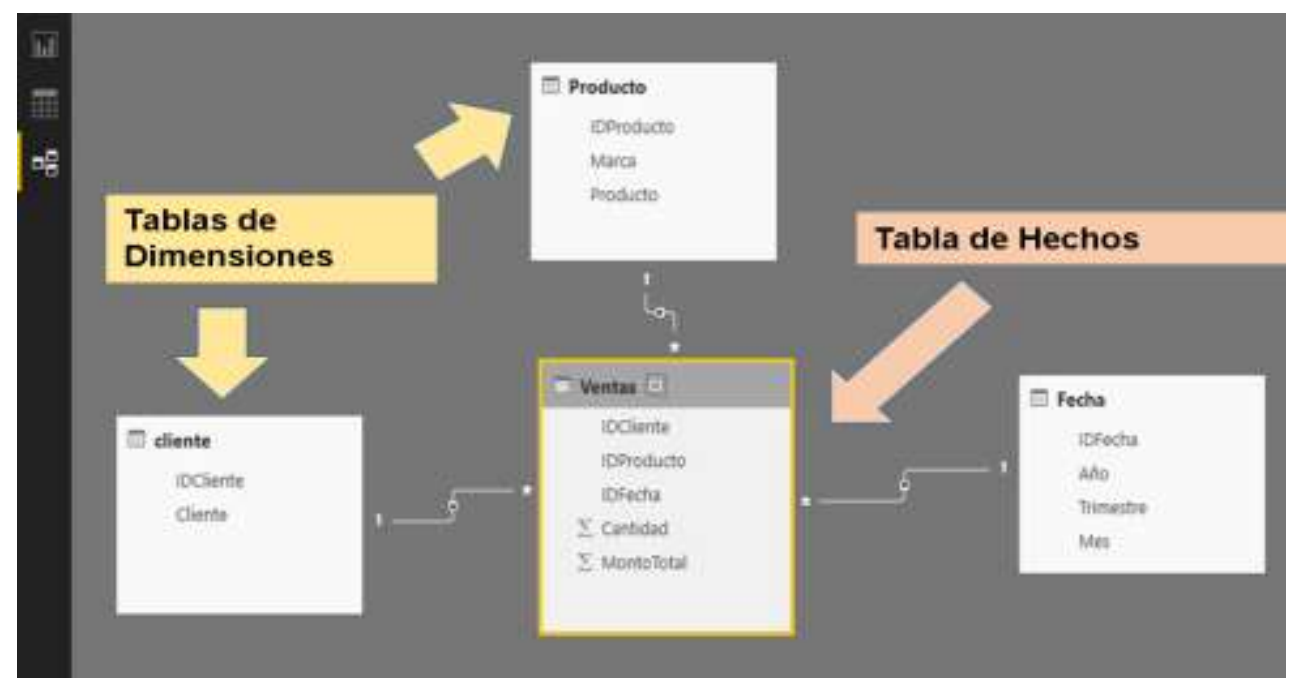

Figura 4. Modelo estrella - Hechos y Dimensiones

\section{Proceso ETL}

El proceso de extracción, transformación se realiza en Microsoft Excel con el propósito de estandarizar la información, es decir que los datos deben ser guardados con coherencia de formato, cambio de unidad, operaciones, etc.; por lo general las transacciones en bruto presentan datos que no interesan o son duplicados, a estos datos se realizar una limpieza o son filtrado, por último, se procede a cargar la base de datos consolidada en los dos programas Power Bl y Access. 


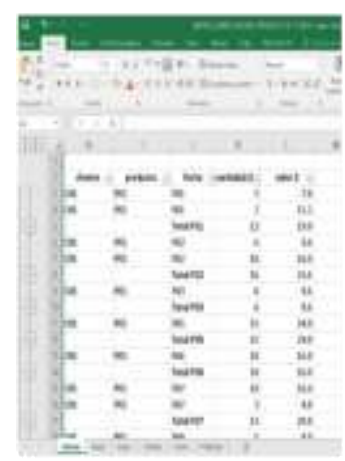

Extracción y transformación en Microsoft Excel
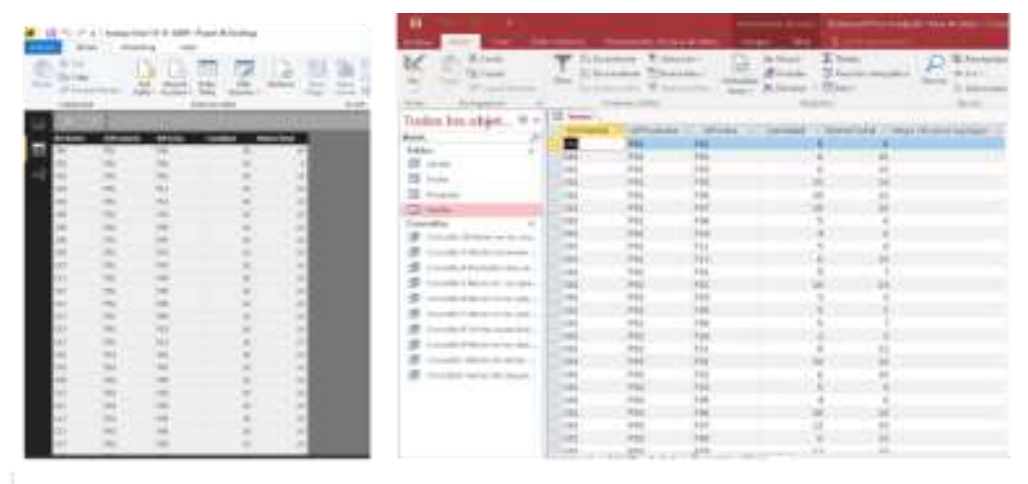

Carga en Power Bl y Microsoft Access

Figura 5. ETL

\section{Data mart}

Para el diseño del Data mart, se realizó con las herramientas Power $\mathrm{BI}$ y Microsoft Access. Los datos se cargaron desde la base de datos de ventas para luego diseñar el modelo estrella y poder generar reportes o consultas; cabe mencionar que para el diseño de la data mart solo se utiliza información específica de un departamento en este caso el área de ventas y se implementa a través de cubos de datos los mismo que son similares a la tabla de hechos y dimensiones.
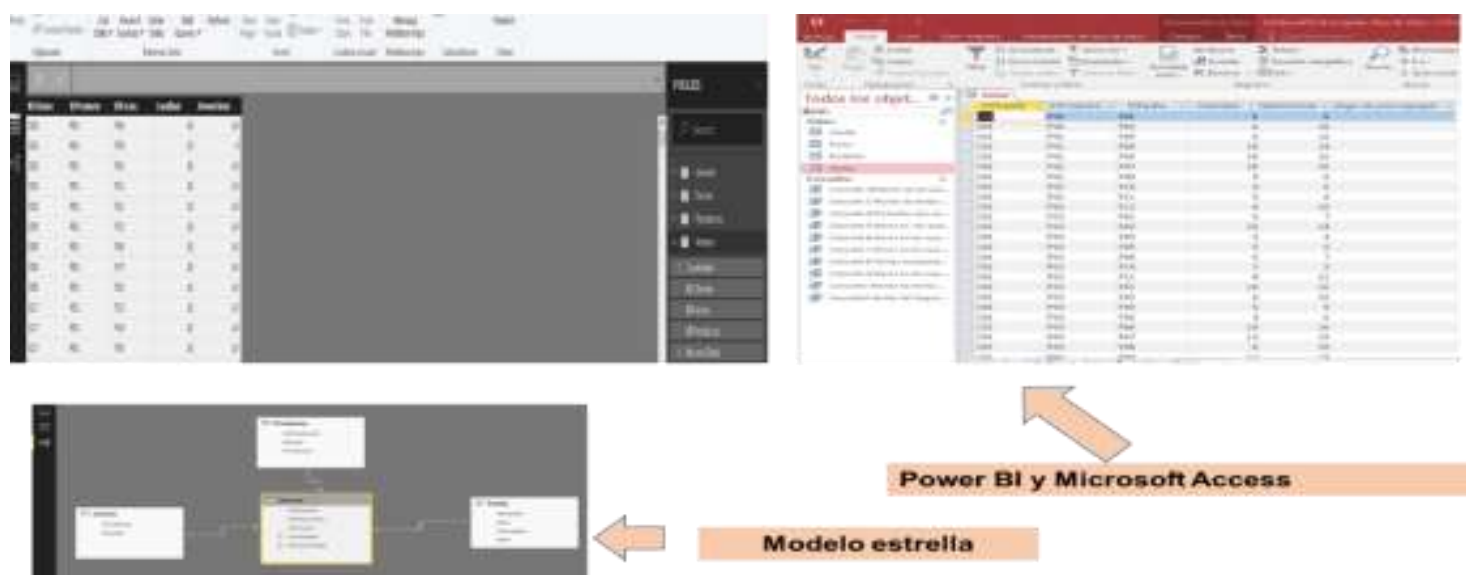

Power Bl y Microsott Access

Modelo estrelfa

Figura 6. Data Marts - Power BI - Access. 


\section{Consultas OLPT (procesamiento transaccional en línea) en Access y Power BI para su visualización}

La herramienta OLPT se podría decir que es un tipo de procesamiento que facilita y administra aplicaciones transaccionales, usualmente para entrada de datos, recuperación y procesamiento de transacciones, es lo que se procede a consultar con el uso de los Softwares como es el Access y Power BI, para su posterior visualización de los resultados.

Consulta N.- 1: Ventas acumuladas en el periodo 2018.

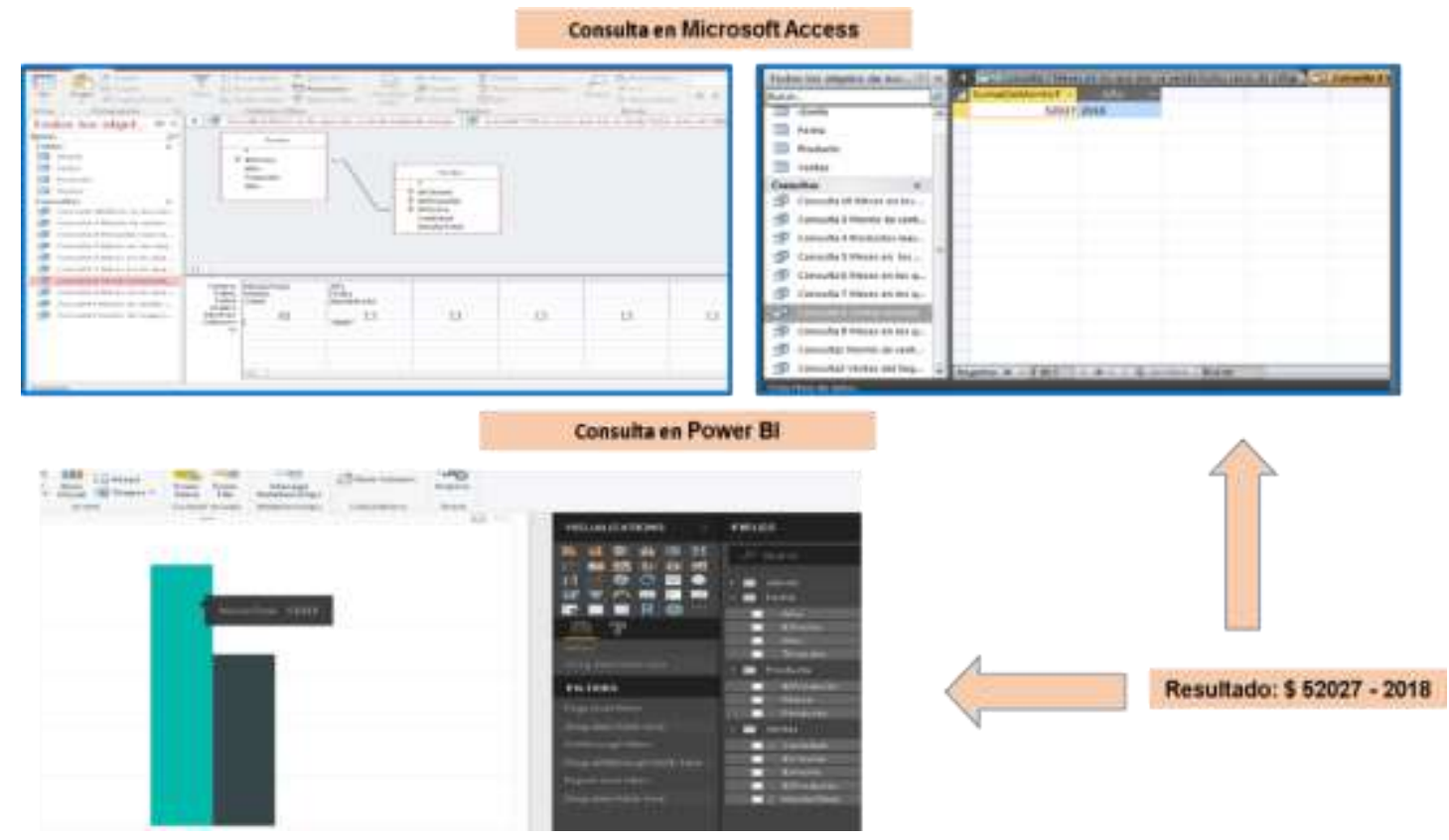

Figura 7. Ventas acumuladas en el periodo 2018 
Revista Arbitrada Interdisciplinaria KOINONIA

Año V. Vol V. N¹0. Julio - Diciembre 2020

Hecho el depósito de Ley: FA2016000010 ISSN: 2542-3088

FUNDACIÓN KOINONIA (F.K). Santa Ana de Coro. Venezuela.

Esteban Ismael Cordero-Naspud; Juan Carlos Erazo-Álvarez; Cecilia Ivonne Narváez-Zurita; Diego Marcelo Cordero-Guzmán

Consulta N.- 2: Monto de ventas en primer trimestre del2018.
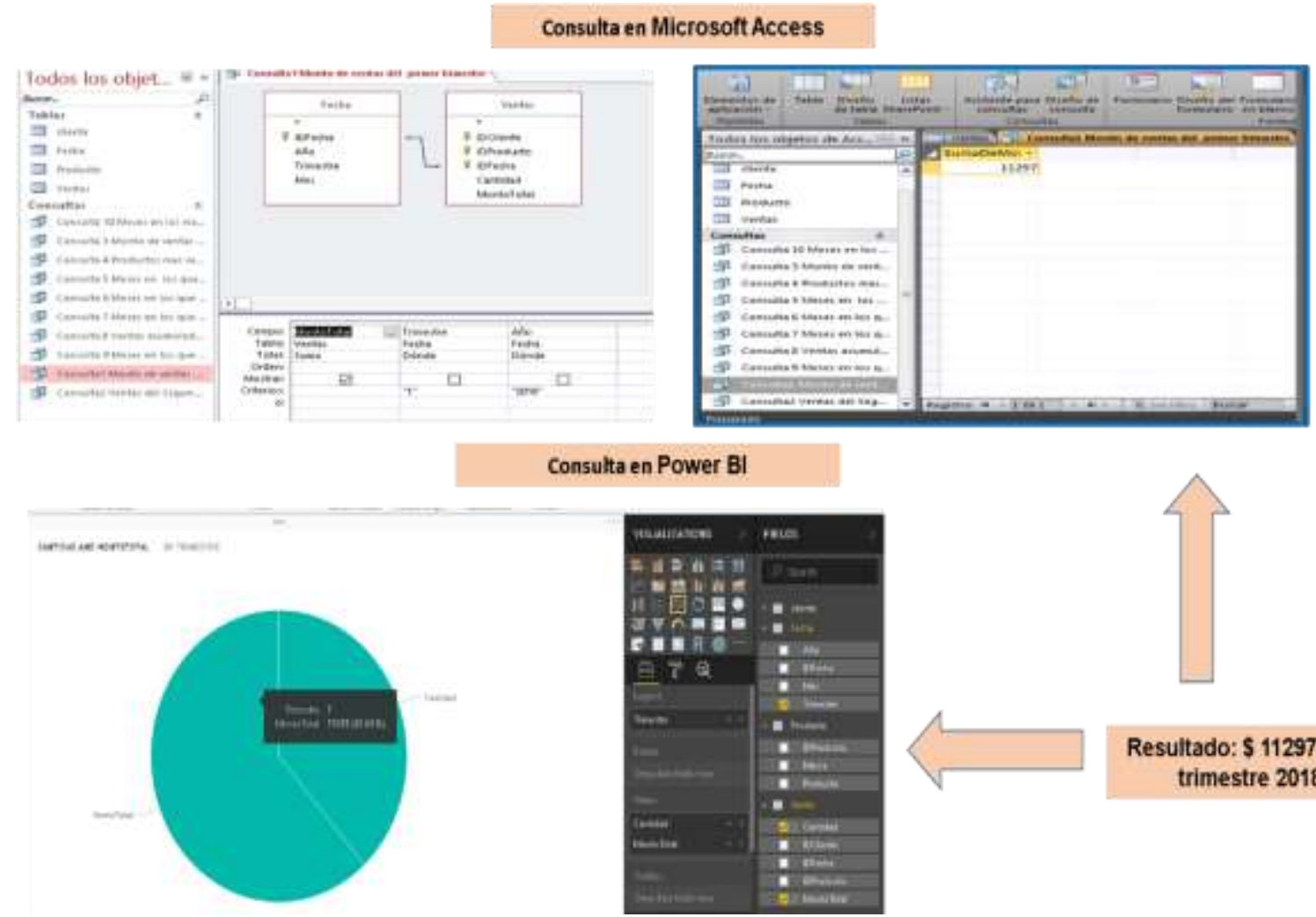

Resultado: \$ 11297 - 1er trimestre 2018

Figura 8. Monto de venta primer trimestre 2018 
Revista Arbitrada Interdisciplinaria KOINONIA

Año V. Vol V. N¹0. Julio - Diciembre 2020

Hecho el depósito de Ley: FA2016000010 ISSN: 2542-3088

FUNDACIÓN KOINONIA (F.K). Santa Ana de Coro. Venezuela.

Esteban Ismael Cordero-Naspud; Juan Carlos Erazo-Álvarez; Cecilia Ivonne Narváez-Zurita; Diego Marcelo Cordero-Guzmán

Consulta N.- 3: Producto más vendido representados en valor monetario

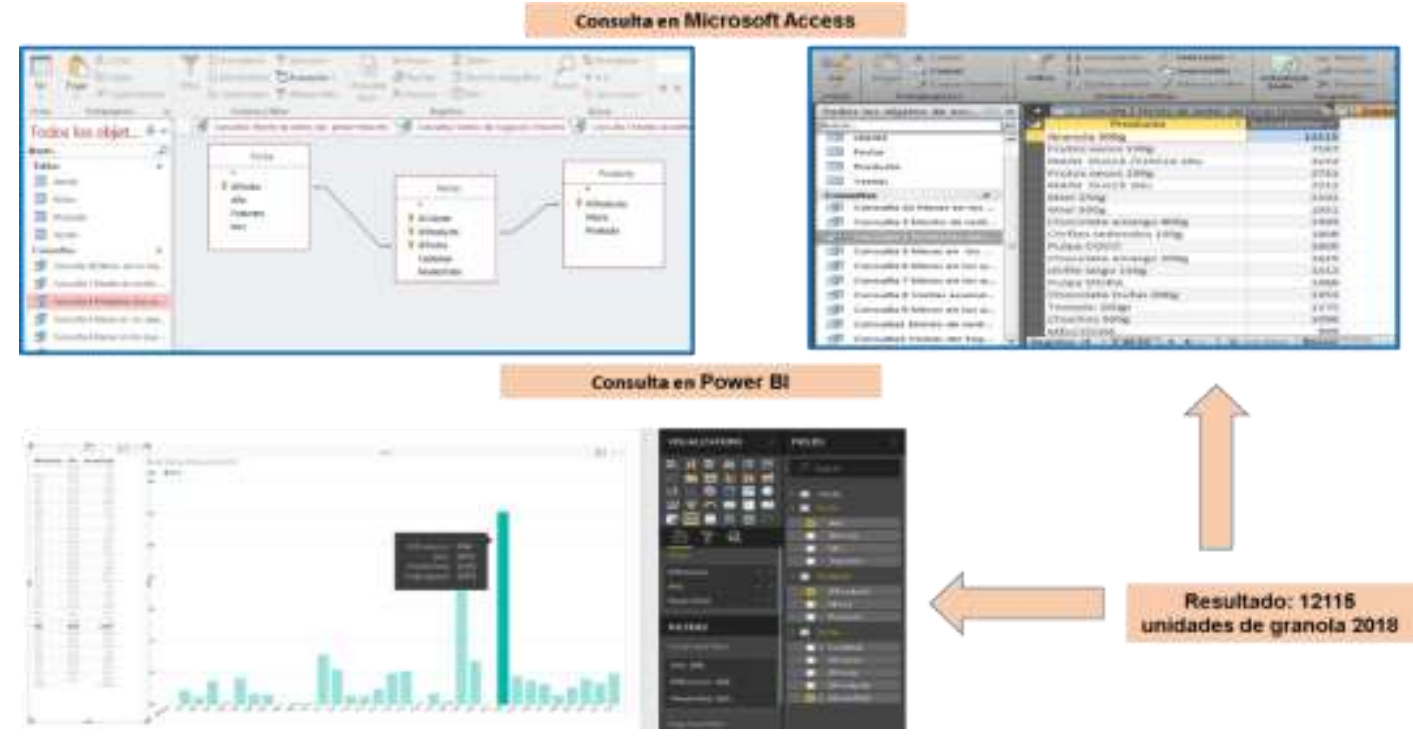

Figura 9. Producto más vendido representado en valor monetario 
Consulta N.- 4: Mes donde existe más rotación de un determinado producto por cliente.
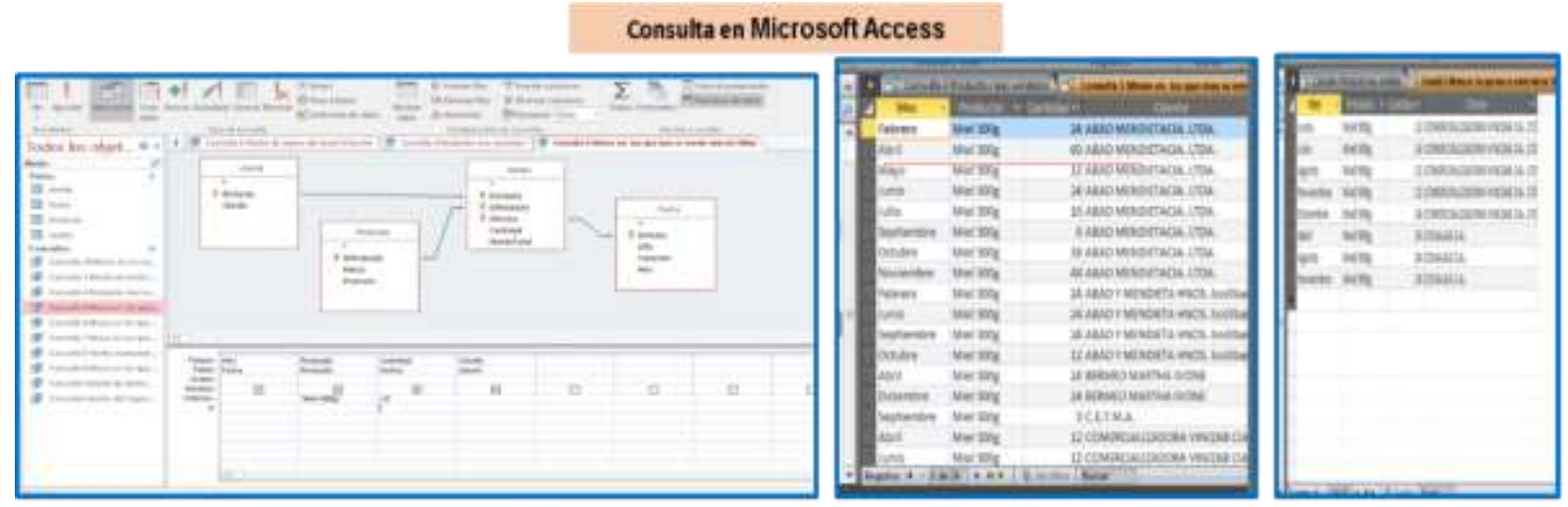

\section{Consulta en Power BI}
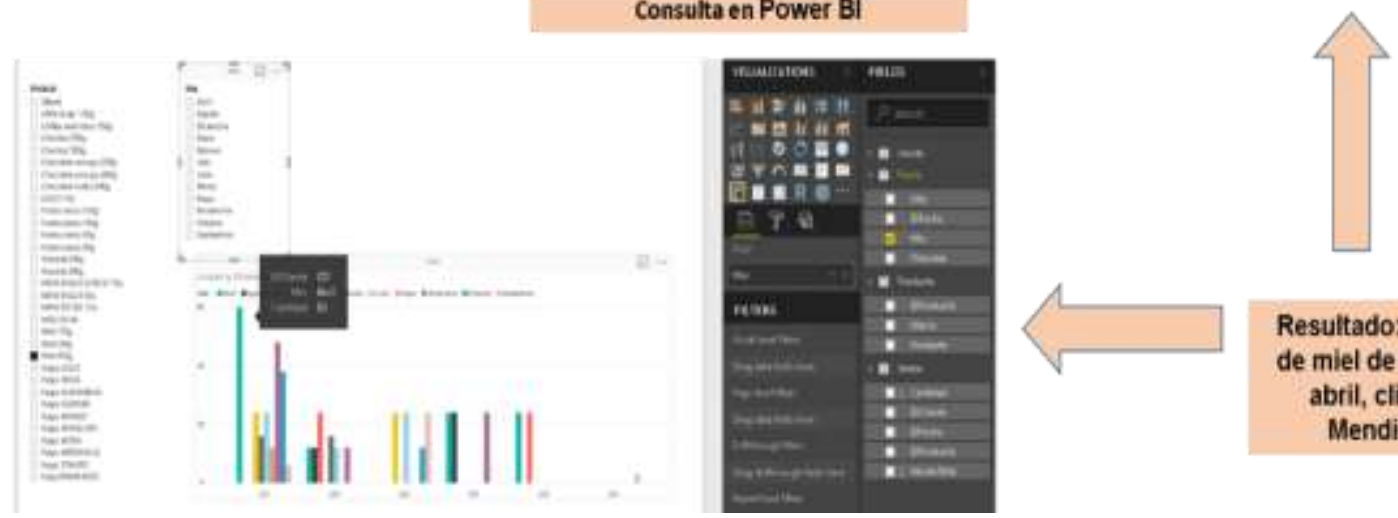

Resultado: 60 unidades de miel de $500 \mathrm{~g}$ mes de abril, cliente Abad Mendieta Ltda.

Figura 10. Mes donde existe mayor rotación de un determinado producto.

Con esta serie de consultas demostramos que la Inteligencia de negocios orientado a las ventas brinda un soporte adecuado para la toma de decisiones, permite contar con información de calidad, reduce costos y automatiza los procesos con la rapidez necesaria que requiere un administrador; en este sentido, al utilizar cualquiera de estos dos programas Power $\mathrm{BI}$ o Access, se puede evidenciar que ofrecen una interfaz muy intuitiva, es decir fácil de manejo para administradores de negocios. 


\section{Esquema de un Tablero de Comandos para el Datamart - ventas}

\section{KPI y diseño de la interfaz gráfica}

Indicador Clave de Desempeño o Medidor de Desempeño, son claves dentro de un negocio, son utilizados y aplicables en cualquier área de negocio o sector productivo, su finalidad es apoyar a tomar mejores decisiones respecto al estado actual de un proceso, proyecto, estrategia y de esta forma, poder definir una línea de acción futura, dicho esto se propone los siguientes indicadores que en un futuro puedan ser utilizados al implementar un sistema $\mathrm{BI}$ en la organización.

Indicadores: A continuación, se presentan varios indicadores financieros que permitirán tomar decisiones oportunas a las pymes.

\section{Tabla 1}

Indicador de Utilidad Bruta

Nombre

Utilidad Bruta

indicador:

$\begin{array}{ll}\text { Definición } & \begin{array}{l}\text { Utilidad bruta es la diferencia entre los ingresos (ventas netas) de la } \\ \text { empresa y el costo de los bienes vendidos. } \\ \text { Formula }\end{array} \\ \text { Cálculo } & \text { UBilidad bruta }=\$ 52.014 .003-\$ 27.687,69=\$ \mathbf{2 4 . 3 2 6 , 3 1} \\ \text { Unidad } & \text { Monto en dólares } \\ \text { Periodicidad } & \text { Anual } \\ \text { Proceso } & \text { Ventas } \\ \text { Responsable } & \text { Departamento Financiero } \\ \text { Interpretación } & \text { La utilidad bruta de la empresa en el año } 2018 \text { luego de restar los } \\ & \text { costos operativos es de 24.325,31. }\end{array}$


Revista Arbitrada Interdisciplinaria KOINONIA

Año V. Vol V. N¹0. Julio - Diciembre 2020

Hecho el depósito de Ley: FA2016000010 ISSN: 2542-3088

FUNDACIÓN KOINONIA (F.K). Santa Ana de Coro. Venezuela.

Esteban Ismael Cordero-Naspud; Juan Carlos Erazo-Álvarez; Cecilia Ivonne Narváez-Zurita; Diego Marcelo Cordero-Guzmán

\section{Tabla 2}

Incremento de facturación mensual

\begin{tabular}{|c|c|}
\hline $\begin{array}{l}\text { Nombre } \\
\text { indicador: }\end{array}$ & Incremento de facturación mensual \\
\hline Definición & $\begin{array}{l}\text { Una de las mediciones más importantes que debe realizar el área de } \\
\text { ventas es el incremento de la facturación mensual. Permite visualizar } \\
\text { mes a mes si aumentan o disminuyen las ventas. }\end{array}$ \\
\hline Formula & $\begin{array}{l}\text { IFM: [(Facturación del mes en curso - Facturación del mes } \\
\text { anterior) / facturación del mes anterior] x } 100\end{array}$ \\
\hline Cálculo & $\mathrm{IFM}: \frac{\$ 5836,75-3945,61}{\$ 3945.61} \times 100=47.93 \%$ \\
\hline Unidad & Porcentaje \\
\hline Periodicidad & Anual \\
\hline Proceso & Ventas \\
\hline Responsable & Departamento Financiero \\
\hline Interpretación & $\begin{array}{l}\text { La empresa tuvo un incremento del } 47.93 \% \text {, comparando los meses } \\
\text { de agosto y septiembre. }\end{array}$ \\
\hline
\end{tabular}

\section{Tabla 3}

Rotación de inventario.

\begin{tabular}{|c|c|}
\hline $\begin{array}{l}\text { Nombre del } \\
\text { indicador: }\end{array}$ & Índice de fidelización \\
\hline Definición: & $\begin{array}{l}\text { Es indicador de rendimiento capaz de medir la rentabilidad del cliente a } \\
\text { lo largo del tiempo, analizando el valor monetario actual y desglosando } \\
\text { la proyección de flujos de caja entre la empresa y el cliente. }\end{array}$ \\
\hline Fórmula: & 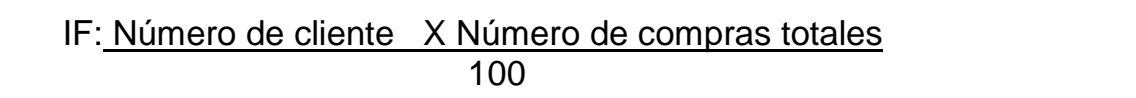 \\
\hline Cálculo: & $\mathrm{IF}: \frac{20 \times 73=14.6 \%}{100}$ \\
\hline Unidad: & Porcentaje \\
\hline Periodicidad: & Anual \\
\hline Proceso: & Ventas \\
\hline Responsable: & Departamento Financiero \\
\hline
\end{tabular}


Interpretación: Esto indica que el índice de fidelización de los clientes es del 14.6\%, esto quiere decir 3 de los 20 clientes de distribuidora tienen lealtad de compra a los productos a la empresa de forma continua o periódica.

\section{Tabla 4}

Volumen de compras por cliente

\section{Nombre del Volumen de compras por clientes}

indicador:

Definición:

$$
\begin{aligned}
& \text { Realizan compras en un volumen que está dentro del promedio } \\
& \begin{array}{l}
\text { general. Por lo general, son clientes que están } \\
\text { empresa, elisfechos con la } \\
\text { realizan compras habituales. }
\end{array}
\end{aligned}
$$

Fórmula:

VCC: Total de compras

Total de clientes

Cálculo:

VCC: $27687,69=\mathbf{1 3 8 4 . 3 8}$

Unidad:

Porcentaje

Periodicidad: Anual

Proceso:

Ventas

Responsable: Departamento Financiero

Interpretación: El volumen de compra por cada cliente es de 1.384 dólares. Esto quiere decir que para cubrir todos los pedidos de los 20 clientes la empresa Cordero deberá comprar por cliente 1.384 dólares. 
Revista Arbitrada Interdisciplinaria KOINONIA

Año V. Vol V. N 10 . Julio - Diciembre 2020

Hecho el depósito de Ley: FA2016000010

ISSN: $2542-3088$

FUNDACIÓN KOINONIA (F.K). Santa Ana de Coro. Venezuela.

Esteban Ismael Cordero-Naspud; Juan Carlos Erazo-Álvarez; Cecilia Ivonne Narváez-Zurita; Diego Marcelo Cordero-Guzmán

\begin{tabular}{|c|c|c|c|c|c|c|c|c|c|c|}
\hline \multirow[b]{2}{*}{ Perspectiva } & \multirow[b]{2}{*}{$\begin{array}{c}\text { Objetivos } \\
\text { Estrategicos }\end{array}$} & \multirow[b]{2}{*}{ Indicadores } & \multirow[b]{2}{*}{ Metas } & \multirow[b]{2}{*}{ Tolerancia } & \multirow[b]{2}{*}{$\begin{array}{c}\text { Desempeño } \\
\text { Real }\end{array}$} & \multicolumn{3}{|c|}{ Rangos } & \multirow[b]{2}{*}{$\begin{array}{c}\text { Areas } \\
\text { Responsables }\end{array}$} & \multirow[b]{2}{*}{ Iniciativa de acción } \\
\hline & & & & & & \begin{tabular}{l|l} 
& \\
$a$ & \\
$j$ \\
0
\end{tabular} & \begin{tabular}{l|} 
\\
$e$ \\
$d$ \\
$d$ \\
$i$ \\
0
\end{tabular} & $\begin{array}{l}0 \\
p \\
t \\
i \\
m \\
0\end{array}$ & & \\
\hline \multirow{4}{*}{$\underset{\substack{\infty \\
\frac{\infty}{c}}}{\stackrel{0}{\infty}}$} & \multirow{4}{*}{$\begin{array}{l}\text { El objetivo es } \\
\text { fortalecer la toma } \\
\text { de decisiones en } \\
\text { el area de ventas }\end{array}$} & Utilidad Bruta & $\$ 30,000.00$ & $\$ 20,000.00$ & $\$ 24.326,31$ & & $\mathbf{x}$ & & $\begin{array}{l}\text { Departamento } \\
\text { de ventas }\end{array}$ & $\begin{array}{l}\text { Relizar mayor publicidad } \\
\text { mediante redes sociales }\end{array}$ \\
\hline & & $\begin{array}{l}\text { Incremento de } \\
\text { Facturación Mensual }\end{array}$ & $60 \%$ & $40 \%$ & $47.93 \%$ & $x$ & & & $\begin{array}{l}\text { Departamento } \\
\text { de ventas }\end{array}$ & $\begin{array}{l}\text { Realizar promociones } \\
\text { de los productos que } \\
\text { tienen menor rotacion }\end{array}$ \\
\hline & & $\begin{array}{l}\text { Indice de } \\
\text { Fidelización }\end{array}$ & $20 \%$ & $10 \%$ & $14.60 \%$ & & & $\mathbf{x}$ & $\begin{array}{l}\text { Departamento } \\
\text { de ventas }\end{array}$ & $\begin{array}{l}\text { Mejorando los precios } \\
\text { para el cliente que } \\
\text { compre en volumen. }\end{array}$ \\
\hline & & $\begin{array}{l}\text { Volumen de } \\
\text { compras por cliente }\end{array}$ & 2000 & 1200 & 1384.38 & & $\mathbf{x}$ & & $\begin{array}{l}\text { Departamento } \\
\text { de ventas }\end{array}$ & $\begin{array}{l}\text { Incentivar la compra de } \\
\text { los clientes otorgan mas } \\
\text { tiempo de pago }\end{array}$ \\
\hline
\end{tabular}

Figura 11. Componentes y subcomponentes del sistema inteligente de negocios

\section{CONCLUSIONES}

La inteligencia de negocios empresarial ofrece beneficios como la capacidad de aprender diferentes facetas de los consumidores y futuros clientes, reduce gastos, acelera la velocidad de estudio, ayuda a crear objetivos prácticos y funciona en la realidad. Por lo tanto, el uso de un Sistema Inteligente de Negocios es importante, de modo que el control de gestión está optimizado para tener un fácil acceso a los datos que permiten a los gerentes de la organización construir predicciones basadas en el conocimiento recopilado y, por lo tanto, determinar posibles estrategias que permitan el crecimiento de la empresa.

Con lo antes expuesto la organización no cuenta con un sistema inteligente, el mismo que ayude a la toma de decisiones empresariales, por cuanto los datos se encuentran dispersos en diferentes hojas de cálculos y a su vez no se trasforma en información de calidad generando un falso conociendo en su entono de negocio; es por eso que se propuso un prototipo de $\mathrm{Bl}$ en el área de ventas para demostrar a la gerencia los beneficios de esta herramienta empresarial; en este sentido se realizaron consultas e indicadores de gestión para el proceso de ventas de la empresa bajo diversas perspectivas que permite un mejor análisis de la información; además mejoró 
significativamente la calidad de la información haciendo más confiables los reportes generados a partir de ella, brindando un soporte para la toma de decisiones en la empresa; al mismo tiempo se propuso indicadores KPI'S de gestión para el proceso de ventas de la empresa con el objetivo de mejorar la toma de decisiones en esta área encaminadas a incrementar la rentabilidad de una empresa.

Con respecto al uso del sistema $\mathrm{BI}$ en otras pymes los resultados proyectaron que varias pymes no cuentan con un sistema inteligente; y las que si manejan BI han optado por utilizar la herramienta del cuadro de mandos integral, en esta razón la cantidad elevada de organizaciones que no utilizan $\mathrm{BI}$ en sus organizaciones nos lleva a creer que las pymes aún no están preparadas para el uso de esta herramienta valiosa, por el temor de arriesgarse a manejar nuevas tecnologías en bien de su negocio o por los alto costo y tiempo que lleva implementar este tipo de SI; resulta para sus propietarios un gasto innecesario para el tipo de negocios ya sea este pequeño o grande, sin saber los altos beneficios que puede generar en la actualidad y futuro.

\section{FINANCIAMIENTO}

No monetario.

\section{AGRADECIMIENTO}

A la Directiva de las pymes de Azogues, provincia del Cañar por el apoyo al desarrollo de la investigación.

\section{REFERENCIAS CONSULTADAS}

Abrego-Almazán, D, Sánchez-Tovar, Y, \& Medina-Quintero, J. (2017). Influencia de los sistemas de información en los resultados organizacionales. [Influence of information systems on organizational results]. Contaduría y administración, 62(2), 303-320. https://dx.doi.org/10.1016/j.cya.2016.07.005 
Camargo-Vega, J. J., Joyanes-Aguilar, L., \& Giraldo-Marín, L. M. (2016). La inteligencia de negocios como una herramienta en la gestión académica. [Business intelligence as a tool in academic management]. Revista Científica, 1(24), 110120. https://doi.org/10.14483/10.14483/udistrital.jour.RC.2016.24.a11

Cordero-Guzmán, D. M., \& Rodríguez López, G. (2017). La inteligencia de negocios: una estrategia para la gestión de las empresas productivas. [Business intelligence: a strategy for the management of productive enterprises]. CIENCIA UNEMI, $\quad 10(23), \quad 40-48 . \quad$ https://doi.org/10.29076/issn.25287737vol10iss23.2017pp40-48p

Coronel, C., Morris , S., \& Rob, P. (2011). Database systems: Design, implementation and management. [Sistema de base de datos: Diseño, implementación y gestión.]. Recuperado de https://n9.cl/i9a58

Da Silva, J., Pastor, A. \& Pastor, J. (2014). El uso del Cuadro de Mando Integral como instrumento de medición para comparar los modelos de Excelencia de Gestión. [The use of the Balanced Scorecard as a measurement instrument to compare the models of Management Excellence. ].Revista Ibero Americana de Estratégia, 13(4), 18-32.

González, J., Palacios, J. \& Perea, J. (2017). La Inteligencia de Negocios y su rol en la agilidad organizacional [Business Intelligence and its role in organizational agility]. Criterio Libre, 15(26), 239-258. Recuperado de https://n9.cl/n9wi

Guajardo-Cantú, G., \& Andrade-De-Guajardo, N. (2014). Contabilidad Finaciera [Financial Accounting]. (Sexta edición ed.). Mexico D.F.: Mac Graw- Hill Interamericana. Recuperado de https://n9.cl/sxal

Gutiérrez-Camelo, A. A., Devia-Llanos, M. A., \& Tarazona-Bermúdez, G. M. (2016). Research inteligencia de negocios: estudio de caso sector tecnológico colombiano. [Business Intelligence Research: Case Study in the Colombian Technology Sector]. Redes De Ingeniería, 7(2), 156-169. https://doi.org/10.14483/udistrital.jour.redes.2016.2.a05

Herazo, J. (2018). Beneficios de la inteligencia de negocios (BI - Business Intelligence) en su empresa. [Benefits of business intelligence (BI - Business Intelligence) in your company]. Recuperado de https://n9.cl/p91j

Instituto Nacional de Estadistica y Censos (2019). Encuesta a empresas. [Business survey]. Recuperado de https://n9.cl/0zz7 
Kaplan, R. S. \& Norton, D. P. (2009). Cuadro de mando integral. [Balanced scorecard]. Barcelona, Gestión 2000.

Koontz, H., Weihrich, H., \& Cannice, M. (2012). Administración, una perspectiva global y empresarial [Administration, a global and business perspective]. Mexico D.F.: McGraw-Hill Educación. Recuperado de https://n9.cl/zxnh4

Laudon, K. \& Laudon, J. (2012). Sistemas de información gerencial [Management information systems]. PEARSON. Recuperado de https://n9.cl/gpqn

Medina-Chicaiza, R., Chiliquinga-Vejar, L., \& Ortiz-Barba, A. (2016). Aproximación sobre la inteligencia de negocios en las PYME. [Approach to business intelligence in SMEs]. Dominio de las Ciencias, 2(4), 370-382. Recuperado de https://n9.cl/8f72

Muñoz, D. (2014). Cuadro de Mando Integral para la Planta Industrializadora de Sal y Alimentos Bolivianos [Balanced Scorecard for the Bolivian Food and Salt Industrializing Plant]. Recuperado de https://n9.cl/bioej. Revista Perspectivas(33), 151-175. Obtenido de http://www.redalyc.org/pdf/4259/425941263005.pdf

Muñoz-Hernández, H., Osorio-Mass, R. C., \& Zúñiga-Pérez, L. M. (2016). Inteligencia de los negocios. Clave del éxito en la era de la información. [Business Intelligence: Key to Success in the Information Age]. Clío América, 10(20), 194211. https://doi.org/10.21676/23897848.1877

Ramírez, J, \& Vega, O (2015). Sistemas de información gerencial e innovacion para el desarrollo de las organizaciones. [Management Information Systems and Innovation for the Development of Organizations]. Télématique, 14(2),201-213. Recuperado de https://n9.cl/i138

Reyes-Dixson, Y., \& Nuñez-Maturel, L. (2015). La inteligencia de negocio como apoyo a la toma de decisiones en el ámbito académico [Business Intelligence as decision making support system in academic environment]. GECONTEC: Revista Internacional De Gestión Del Conocimiento Y La Tecnología, 3(2), 63-73. Recuperado de https://n9.cl/u02b

Rifai, F. (2015). The knowing-doing-gap and the role of the entrepreneurial university in the development of the knowledge economy. [La brecha entre el saber hacer y el papel de la universidad emprendedora en el desarrollo de la economía del conocimiento]. European Journal of Business and Innovation Research, 4(1), 117. Recuperado de https://n9.cl/b7u20 
Roo-Huerta, A. \& Boscán-Romero, N. (2012). Inteligencia de negocios en la banca nacional: Un enfoque basado en herramientas analíticas [Business Intelligence in National Banking: An Approach Based on Analytical Tools]. Revista Venezolana de Gerencia, 17(59), 548-563. Recuperado de https://n9.cl/jaxm

Saquicela-Rodas, V., Erazo-Álvarez, J., \& Narváez-Zurita, C. (2019). Modelo de Gestión estratégica para las Mipymes que conforman el Pasaje Artesanal Azuayo. [Strategic Management Model for MSMEs that form the Azuayo Artisanal Passage]. Revista Arbitrada Interdisciplinaria Koinonía, 4(1), 206-230. http://dx.doi.org/10.35381/r.k.v4i1.456

Sherman, R. (2014). Business Intelligence Guidebook: From Data Integration to Analytics. [Guía de inteligencia: desde la integración de la data a lo analítico.]. (Edición Kindle ed.).

Teruel, S. (2019). Las 8 etapas en el proceso de toma de decisiones de la empresa. [The 8 stages in the company's decision-making process]. Recuperado de https://n9.cl/45y0

Turban, E., Volonino, L. \& Wood, R. (2013). Information technology for management: Advancing in the sustainable and profitable business growth. [Tecnología de la información para la gestión: avanzar en el crecimiento empresarial sostenible y rentable]. (Novena edición ed.). USA: John Wiley \& Sons. Recuperado de https://n9.cl/daj34

Uvidia-Fassler, M., Cisneros-Barahona, A., Méndez-Naranjo, P., \& Villa-Yánez, H. (2019). Minería de datos para la toma de decisiones en la unidad de nivelación y admisión universitaria ecuatoriana. [Data mining for decision-making in the Ecuadorian university admission and leveling unit]. Cumbres, 4(2), 55-67. Recuperado de https://n9.cl/fh7e9

Vanegas-Lago, E. \& Guerra-Cantera, L. (2013). Sistema de inteligencia de negocios para el apoyo al proceso de toma de desiciones [Business intelligence system to support the decision-making process]. Revista Ingenieria UC, 20(3), 25-34. Recuperado de https://n9.cl/60ej

Vásquez, E. (2015). Minería de datos para la inteligencia de negocios. [data mining for business intelligence]. Recuperado de https://n9.cl/s0n5

Vercellis, C. (2009). Business Intelligence: Data Mining and Optimization for Decision Making. [Inteligencia empresarial: minería de datos y optimización para la toma de decisiones]. John Wiley \& Sons Ltd. 
Revista Arbitrada Interdisciplinaria KOINONIA

Año V. Vol V. $\mathrm{N}^{\circ} 10$. Julio - Diciembre 2020

Hecho el depósito de Ley: FA2016000010

ISSN: 2542-3088

FUNDACIÓN KOINONIA (F.K). Santa Ana de Coro. Venezuela.

Esteban Ismael Cordero-Naspud; Juan Carlos Erazo-Álvarez; Cecilia Ivonne Narváez-Zurita; Diego Marcelo Cordero-Guzmán

@2020 por los autores. Este artículo es de acceso abierto y distribuido según los términos y condiciones de la licencia Creative Commons Atribución-NoComercial-Compartirlgual 4.0 Internacional (CC BY-NC-SA 4.0)

(https://creativecommons.org/licenses/by-nc-sa/4.0/2. 